\title{
Logical Reasoning beyond Classical Logic: An Illustration with Pythagoras Theorem
}

\author{
Francisco Vargas ${ }^{1,2^{*}}$, Keith Stenning ${ }^{3}$ \\ ${ }^{1}$ Universidad el Bosque, Bogotá, COLOMBIA \\ ${ }^{2}$ Ludwigsburg University of Education, Ludwigsburg, GERMANY \\ ${ }^{3}$ University of Edinburgh, UK \\ *CORRESPONDENCE: $\triangle$ fjvargasm@unbosque.edu.co
}

\begin{abstract}
We report on a study conceived with the idea that the use of logic in regard to mathematical reasoning as it actually occurs in practice is not limited to its prominent role in formal deductions and proofs. Interpretation of different mathematical situations elicits in fact the use of mostly unconscious forms of reasoning, close to those of narrative processing, which do not coincide with the expectations of traditional logic. This is pervasive, in particular, in educational situations at different levels, as we illustrate with interpretations which can emerge alongside an apparently obvious mathematical statement, namely, Pythagoras Theorem. We defend the position that analyses of "errors", should start by understanding their prevalence and non arbitrariness. Accordingly, we use a nonclassical logics whose features may give new insights to the kind of learning obstacles often found in the literature, as well as in our results.
\end{abstract}

Keywords: reasoning, logic, conditionals, closed world reasoning, negation as failure

\section{INTRODUCTION}

During the last fifty years the psychological literature on reasoning has provided us, as one of its characteristic themes, a whole body of evidence that the interpretation and use of the logical connectives in different contexts is far from obvious. An important example is the meaning of conditional statements. One of the effects most widely reported and studied is that these are interpreted in different ways, very commonly in disagreement with the meaning of the so called material implication. Well known experiments like the Wason Selection task (Wason, 1968) give us a complex vision that has been widely seen as a lack of the normative logical competency and even as supporting our lack of rationality. Similar trends are present in the educational literature on reasoning in mathematics where we can see that deviations from the standards of traditional logic are commonly understood as mistakes to be eliminated, in accordance with the developmental stage acquired by individuals (see e.g., O’Brien et al., 1971) or through explicit training on logic (Durand-Guerrier et al., 2011).

It is also possible, however, to take into consideration in this context different logical standards and accounts others than classical logic, as has been considered and studied in cognitive psychology (Stenning \& van Lambalgen, 2008). We can see similar proposals for considering alternative interpretations or logics not necessarily regarded as errors in Hoyles and Küchemann (2002) and in D'Amore (2005). Here we propose yet other considerations from developments in logic and AI. These, we claim, may not only help us to reevaluate traditional views on logic, but also on actual human reasoning, providing both a description, and a deeper understanding of it, which is educationally important as a basis for communicating with students. In this

Article History: Received 16 May $2019 \bullet$ Revised 17 July $2019 \bullet$ Accepted 17 July 2019

(C) 2020 by the authors; licensee Modestum Ltd., UK. Open Access terms of the Creative Commons Attribution 4.0 International License (http://creativecommons.org/licenses/by/4.0/) apply. The license permits unrestricted use, distribution, and reproduction in any medium, on the condition that users give exact credit to the original author(s) and the source, provide a link to the Creative Commons license, and indicate if they made any changes. 
paper we report on an experiment about the understanding of a particular conditional statement (Pythagoras' Theorem). The meaning of this statement is apparently obvious (who doesn't know what this theorem is about at an undergraduate level?). Even so, we will see that the meaning of the statement may be underdetermined if we consider what we may take it to imply, and how different interpretations may emerge.

Our results, in part, extend to a different sample and a different instrument already documented results (e.g. in Durand-Guerrier, 2003; Hoyles \& Küchemann, 2002; O’Brien et al., 1971) about how students interpret the implication. We intended to test these and other phenomena (1) at a university level with a students who had received previously some training in traditional logic (even if no mention of logic was made during the test) and (2) with mathematical content where their knowledge was beyond dispute. We were interested in (1) in order to confirm the robustness of the results (which reveal reasoning tendencies which are not modified in general after traditional logic courses). On the other hand, (2) could provide us data on the logical reasoning processes in themselves, reducing possible interference with insufficient or possibly mistaken mathematical knowledge.

As for the analyses, we will use Logic Programming (LP) (a nonmonotonic logic), some of whose salient relevant features we will briefly present. In this choice, we follow some recent trends in the psychology of reasoning (Stenning \& van Lambalgen, 2008), which show that the traditional monolithic identification of logic as classical logic can be widened to the use of other logics more suitable to model and explain the results of a series of psychological experiments.

Logic, we shall argue, is not fixed, given from the outset, but obtained only at the end of an interpretational process. Only once the kind of logic is established can we go beyond the information given and establish a whole model or "world". In this, statements are not taken in isolation, but as part of a whole web of beliefs. This knowledge coordination, being largely unconscious, may lead us to believe and to act in discrepancy from standards acquired or expected through instruction.

\section{METHODS}

The experiment here reported is part of a doctoral thesis on reasoning in Mathematics from an educational perspective, and the use of different logics as means for modelling it. The Participants were 178 nd year Engineering students (mean age $=19$ years) from El Bosque University at Bogotá. All the participants had taken in their first year an introductory course on logic which included classical connectives, truth tables and classical deduction schemas as modus ponens and modus tollens (see below).

\section{Materials}

We applied 40 minute questionnaires about different subjects of elementary mathematics. The following are the translations (originals in Spanish) of the two questions analysed here:

\section{Question P1}

Pythagoras's Theorem may be stated as:

If we have a right-angled triangle with hypotenuse $a$ and legs $b$ and $c$, then the relation $a^{2}=b^{2}+c^{2}$ holds.

As an immediate application of this statement (i.e., not assuming other geometrical facts) we can conclude that:

- The right-angled triangle with $b=1$ and $c=2$ has $a=\sqrt{5}$

- The triangle with $a=4, b=3$ and $c=3$ is not right-angled

- The triangle with $a=5, b=4$ and $c=3$ is right-angled

- If triangle with $b=1$ and $c=2$ is not right-angled then $a$ cannot be $\sqrt{5}$

- The right-angled triangle with $b=3$ and $c=3$ has $a=2 \sqrt{3}$.

Select the option or options that you consider to be correct. If you want to specify something about your selection please do.

\section{Question P2}

For every right-angled triangle with hypotenuse $a$ and legs $b$ and $c$, the following holds (choose a unique option):

- The sum of the areas of the equilateral triangles constructed on the sides $b$ and $c$ equals the area of the equilateral triangle constructed on $a$. 
- The sum of the areas of the semicircles with diameters $b$ and $c$ is equal to the area of the semicircle with diameter $a$.

- The sum of the halves of the areas of the squares constructed on the sides $b$ and $c$ is equal to half the area of the square constructed on $a$.

- The sum of the areas of regular pentagons constructed on the sides $b$ and $c$ is equal to the area of the regular pentagon constructed on the side $a$.

- All the above

- None of the above

Justify briefly your selection.

In Question P1, as may be noticed, the first four options in the menu correspond, in order (the order on the used booklets was randomized), to the schemas modus ponens (MP), modus tollens (MT), affirmation of the consequent $(\mathrm{AC})$ and denial of the antecedent (DA). Formally, the schemas correspond to:

$$
\begin{aligned}
& A \rightarrow B, A \vdash B(\mathrm{MP}) \\
& A \rightarrow B, \neg B \vdash \neg A(\mathrm{MT}) \\
& A \rightarrow B, B \vdash A(\mathrm{AC}) \\
& A \rightarrow B, \neg A \vdash \neg B(\mathrm{DA})
\end{aligned}
$$

A more detailed examination would require the use of predicate logic, for we are dealing in $\mathrm{P} 1$ with a general statement (the theorem) and particular instantiations of it. Nevertheless, we will limit ourselves to propositional logic for the level of analysis required here. As is well known, the first two deductive schemas are classically valid. On the contrary, AC and DA are usually considered as "fallacious" inferences: from the truth of the premisses the truth of the conclusion doesn't necessarily follow. The 5th option in the menu of answers is a filler where application of Pythagoras' Theorem would lead (by MP) to a result different from the proposed $a=2 \sqrt{3}$.

Question P2, on the other hand, is intended to explore how far the students are open to accepting the possibility of an analogy or a generalisation of Pythagoras' Theorem. How closed are they on the already established knowledge? Technically speaking, this will be linked to what is known as closed world reasoning (see below).

\section{RESULTS}

\section{Question P1}

Table 1 summarises the endorsement rates of the 4 deductive schemas already described. The format and the mathematical content of our question make it not directly comparable with the meta-analysis, based on 11 studies, from Evans et al. (1993). Even so, for the sake of reference, we repeat those results here for convenience.

\section{Question P2}

In Table 2 we report the percentages of the chosen option in P2. A generalisation of Pythagoras' Theorem is definitely not a standard scholarly topic in Colombia, so presumably options 1 to 4 were not known to be true by our participants. This is confirmed by the justifications provided by them, almost all of them either absent (36.5\%) or incorrect (53.4\%). Only a few students provided a valid argument for option 3, for which a proof could be directly provided. Our interest focuses though on a qualitative analysis for justifications to option 6.

Table 1. Endorsement rates for deductive schemas in Question P1 in the literature and in our study (N=178)

\begin{tabular}{ccc}
\hline Schemas & Literature & Experiment \\
\hline MP & $89-100 \%$ & $86 \%$ \\
\hline MT & $41-81 \%$ & $50 \%$ \\
\hline AC & $23-75 \%$ & $68 \%$ \\
\hline DA & $17-73 \%$ & $42 \%$ \\
\hline Filler & & $12 \%$ \\
\hline
\end{tabular}


Table 2. Endorsement rates for the menu options in question P2 (N=178)

\begin{tabular}{cc}
\hline Option & Percent \\
\hline 1 & $19.1 \%$ \\
\hline 2 & $3.4 \%$ \\
\hline 3 & $23 \%$ \\
\hline 4 & $0.6 \%$ \\
\hline 5 & 13.5 \\
\hline 6 & 29.2 \\
\hline NA & 11.2 \\
\hline
\end{tabular}

The kind of argumentation that some of our students explicitly follow exhibits a case of negation as failure (NAF), i.e., the assumption that something must be false from the fact that it's not known (in context) to be true. Here, options 1-4 must be false because none of them is included in the known information (basically Pythagora's Theorem). In other words, Pythagoras' Theorem is not only something true, but the whole truth, about figures constructed on the sides of straight angled triangles. We illustrate that NAF may be operating with three of the justifications explicitly provided:

According to Student 1, "You must form squares on b and c in order to obtain that the sum of their areas equals that of the square formed on a" (our emphasis). Similarly, Student 2 affirms: "In question P1 we are told what Pythagoras' Theorem says... this means that it's not applicable for any other answer, for they talk about other geometric figures." (our emphasis). Finally, in Student 3 we find: "Theorems (in the menu) are false given that none of them is related to those concerning right-angled triangles".

\section{SOME TOOLS FROM LOGIC}

20th century development of Logic has provided an enormous variety of logics available for the purposes at hand in different disciplines. This plurality of possibilities allow us to give formal accounts of human reasoning according to the particular situation at hand. Logic is not fixed, doesn't work context-independently, but only through the mediation of a process of interpretation which pragmatically determines it.

Logic Programming (LP) in particular, occupies in our view a special place: born in AI, it was designed for a technical treatment of the problem of planning in environments with incomplete information. This is precisely the problem that human beings face most of the time in real situations that call for reasoning. The fact of taking into account new information throughout the planning process makes LP a 'logic of the process of constructing interpretations for new information'.

We will limit ourselves here to summarising some of the features of LP applicable to the study of human reasoning (a technical treatment may be found in (Doets, 1994)). We mention first two properties relevant for our study:

Syntax: Formulas in LP are restricted to Horn clauses (a conditional with the antecedent being a conjunction of literals and the consequent a single literal). This decreases substantially computational complexity in comparison with classical logic, but LP is still expressive enough to cover significant fragments of human reasoning and language processing, specially if considering the predicate and not only the propositional versions of LP, see Kowalski (2011), Stenning and van Lambalgen (2008) and van Lambalgen and Hamm (2005).

Closed world reasoning: As already mentioned databases or "programs", i.e., sets of Horn clauses, codify the available information. From these, it is possible to extract additional information in a way which substantially differs from classical logic. Particularly important is what is known as "closed world reasoning" (CWR), which essentially manages the problem of overcoming the incompleteness information, treating the database as if it were complete. This assumption is manifested at different levels, e.g. in the treatment of negation. As stated before, the negation as failure (NAF) interpretation assumes that a statement is false if we don't know that it is true (in current context), which means here that it does not belong to the working database.

Another important aspect of CWR is the "completion semantics". Roughly speaking, if the database or program is taken as if complete, the set of all the program clauses with the same consequent is a disjunctive definition of this consequent. In this sense, implications are "biconditionals in disguise" (Kowalski, 2011). 
We mention two other features that we will not use in our discussion, but that we consider important in the context of math education:

Non-monotonicity: The information acquired in a particular situation is treated as belonging to a database. New information may be incorporated into it, which can defeat previously valid conclusions. This makes this logic non-monotonic, a feature that we will not consider in our examples but that makes these logics particularly tuned to human reasoning to interpretations. In particular, this constitutes the very core of what is expected from education: the acquisition of new knowledge may defeat previously supported conclusions and reshape our view on a particular topic.

Inferential character of implication: Classical connectives are truth functional: the truth value of a formula involving them is completely determined by the truth values of the atomic formulas appearing in it. Conditionals in LP behave differently, more as a 'license for inference' in logic programs-a sort of contentful inference rule. The main difference between the two interpretations is the case when the antecedent is known to be false. According to classical logic this makes the conditional true, but in the second case it simply deactivates inferences from the conditional. As noticed by Hoyles and Küchemann (2002) evidence indicates that the last sense seems closer to the interpretation of students in mathematical contexts.

\section{DISCUSSION AND CONCLUSIONS}

The prevalence of misconceptions like the fact that a mathematical statement is often taken as saying "the same thing" as its converse (Hoyles \& Küchemann, 2002) calls for a cognitive account which explains why we reason this way. This is even more necessary considering the fact that this phenomenon is not limited to particular groups or ages: as confirmed again by the results of Question P1, it is present also at a pre-graduate level (even after explicit instruction in logic) with items with mathematical content. The fact that from Pythagoras' Theorem students infer its converse is shown by the high rate of participants that endorse the validity of the option which corresponds to the classically invalid AC schema (68\%). This endorsement rate is, remarkably, even higher in our results than the classically valid MT. The converse of Pythagoras' Theorem is actually valid: Euclid himself demonstrates it as a corollary immediately after the direct theorem. But our participants didn't know this fact: it was not for them a studied result, and when asked in a final test question about their previous knowledge of it, they denied it, and sometimes, again, they even seemed to be unable to distinguish between the two statements. A form of the LP completion semantics, as already shown e.g. in (Stenning \& van Lambalgen, 2008) for similar phenomena, can give a formal account for this "biconditional effect" which has been widely noted in the psychology of reasoning.

Results of Question P2, on the other hand, show us also another form of CWR: What is not known, or has not been explicit asserted, is simply false. Participants argumentation, above, basically asserts that Pythagoras' Theorem cannot be generalised to other figures because what is not explicitly stated in it cannot be true. Here, we emphasise in this phenomenon the underlying logical process using the technical term, NAF, but it can be stated in other forms. It can be approached in terms of informativeness (Grice, 1975): if more informative versions of the theorem are possible (a student may ask) why are they never mentioned by teachers or by standard books? In yet another tradition, psychologist Daniel Kahneman (2011), has coined the term WYSIATI "What You See Is All There Is". Here we want to emphasise, not primarily the "biased" character of this reasoning procedure, but its computational advantages: it allows us to overcome the absence of information by establishing default assumptions about it. We reason as if we have complete information precisely because we don't have it but at the same time, in the real world, we need to infer, decide, and act in finite time overcoming this incompleteness. Of course, a converse of a theorem should not be inferred automatically without proof (as in P1), and theorems going beyond the established ones are not necessarily false (as seen in P2), and we are not doubting that our students should acknowledge this. What we want to stress is that what they are applying is not necessarily per se irrational. What all too often is required from us in everyday situations is what mobilises our reasoning in situations such as those presented.

In this sense, we think that characterisations for these phenomena as "child logic" , in contrast to a "math logic" (O'Brien, et al., 1971) may be misleading in several ways: on one hand, treating this form of reasoning as a "poor man's logic" in the absence of full acquisition of classical logic, and not as a phenomenon that can be characterized, explained, and even justified on its own. On the other hand, the use of this kind of logic is not at all restricted to a certain developmental stage as this name ("child") seems to indicate. It may well be thought as a fundamental component of mature thought, and even of mathematical thought in its heuristic informal aspects. We want to replace the "poor man's logic" attitude on the part of the teacher, with a "two 
logics working together" attitude. And to raise the educational question whether explicitly teaching that there are "two logics" may actually be a good teaching strategy at some levels.

Both questions, P1 and P2 indicate that in interpreting a statement we tend to establish not punctual facts, but a surrounding environment, a connected "web of beliefs", a "world", which is often, in the technical sense here specified, a closed world. In both cases this allows us to infer something not explicitly known, studied or affirmed: in the first case, positively, an additional implication is taken for granted, in the second case, negatively, it is taken for granted that what is not in the theorem is not true.

This certainly connects to what Fischbein (1987) already identified about intuition. According to him, in this "crystallised-very often prematurely closed-conception... incompleteness or vagueness of information is masked by special mechanisms for producing the feelings of immediacy, coherence and confidence". Intuitions, in addition, "cannot be treated effectively and positively as mere isolated symptoms but rather as manifestations of highly articulated and very complex structures". What we previously sketched, is an attempt to describe part of this articulation of our mathematical knowledge as it occurs in practice.

The phenomena described are not something circumstantial or accidental: they lie at the ground of very common "mistakes", as those documented in the literature, but possibly also of ways of thought that are adequate, useful and absolutely necessary in other circumstances that require intelligence, either human or artificial (Kowalski, 2011). We propose these turn out to be completely fundamental and indispensable faced by the problem of processing information in order to construct new interpretations, which would explain the robustness of the phenomena. We are in front of a genuine epistemological obstacle (Brousseau, 1997) that to be properly overcome, calls for a description of what are the processes so commonly at play, and a comprehension that there is an inner logic behind them (Stenning \& van Lambalgen, 2008). This could lead us eventually not only to an explanation of why it is so, but to more awareness of the strategies for dealing with the reality of our cognition.

\section{ACKNOWLEDGEMENT}

The authors thank Laura Martignon for her helpful comments on various aspects of this paper.

\section{Disclosure statement}

No potential conflict of interest was reported by the authors.

\section{Notes on contributors}

Francisco Javier Vargas - Universidad el Bosque, Bogotá, Colombia and Ludwigsburg University of Education, Ludwigsburg, Germany.

Keith Stenning - University of Edinburgh, UK.

\section{REFERENCES}

Brousseau, G. (1997). Theory of Didactical Situations in Mathematics. Netherlands: Kluwer.

D'Amore, B. (2005). Secondary school students' mathematical argumentation and Indian Logic (Nyaya). For the learning of mathematics, 25(2), 26-32.

Doets, K. (1994). From Logic to Logic Programming. Cambridge, MA: MIT Press. https://doi.org/10.7551/mitpress/3133.001.0001

Durand-Guerrier, V. (2003). Which notion of implication is the right one? From logical considerations to a didactic perspective. Educational Studies in Mathematics, 53, 5-34. https://doi.org/10.1023/A:1024661004375

Durand-Guerrier, V., Boero, P., Douek, N., Epp, S. S., \& Tanguay, D. (2011). Examining the role of logic in teaching proof. In Proof and proving in mathematics education (pp. 369-389). Springer Netherlands. https://doi.org/10.1007/978-94-007-2129-6_16

Evans, J. S. B., Newstead, S. E., \& Byrne, R. M. (1993). Human reasoning: The psychology of deduction. Psychology Press.

Fischbein, H. (1987). Intuition in science and mathematics: An educational approach (Vol. 5). Springer Science \& Business Media. 
Grice H. P. (1975). Logic and conversation. In P. Cole \& J. Morgan (Eds.), Syntax and Semantics: Speech Acts, volume 3. London: Academic Press.

Hoyles, C., \& Küchemann D. (2002). Students' understanding of logical implication. Educational Studies in Mathematics, 51, 193-223. https://doi.org/10.1023/A:1023629608614

Kahneman, D. (2011). Thinking, fast and slow. Macmillan.

Kowalski, R. (2011) Computational Logic and Human Thinking: How to be Artificially Intelligent. Cambridge: Cambridge University Press. https://doi.org/10.1017/CBO9780511984747

O’Brien, T. C., Shapiro, B. J., \& Reali, N. C. (1971), Logical thinking - language and context, Educational Studies in Mathematics, 4, 201-219. https://doi.org/10.1007/BF00303385

Stenning, K., \& van Lambalgen, M. (2008). Human Reasoning and Cognitive Science. Cambridge, MA: MIT Press. https://doi.org/10.7551/mitpress/7964.001.0001

Van Lambalgen, M., \& Hamm, F. (2005). The proper treatment of events. New Jersey, USA: Blackwell Publishing. https://doi.org/10.1002/9780470759257

Wason, P. C. (1968). Reasoning about a rule. Quarterly Journal of Experimental Psychology, 20, $273-281$. https://doi.org/10.1080/14640746808400161

http://www.iejme.com 